\title{
ESTABILIZADOR NEURAL NÃO-LINEAR PARA SISTEMAS DE POTÊNCIA TREINADO POR REDE DE CONTROLADORES LINEARES
}

\author{
José A. L. Barreiros* \\ barreiro@ufpa.br \\ Walter Barra Jr.* \\ walbarra@ufpa.br
}

André M. D. Ferreira ${ }^{\dagger}$

andre@amazon.com.br

Carlos Tavares-da-Costa Jr.*

cartav@ufpa.br

\author{
Rafael Suzuki Bayma* \\ rafael_bayma@yahoo.com.br
}

*Universidade Federal do Pará (UFPA), Departamento de Engenharia Elétrica e de Computação (DEEC)

Campus Universitário do Guamá, Belém, PA, 66075-900, Fone/Fax: (91) 3201-7680

${ }^{\dagger}$ Centro Federal de Educação Tecnológica do Pará (CEFET-PA), Diretoria de Ensino (DE), Curso de Eletrotécnica

Av. Almirante Barroso, 1155 (Marco), Belém, PA, 66093-020, Fone: (91) 3201-1764

\begin{abstract}
Power System Stabilizers (PSS) have been applied as the most common solution to damp small magnitude and low frequency oscillations in modern electric power systems. Conventional Stabilizers, with fixed structure and parameters, have been used with this objective for several decades, but there are some system operation conditions where the performance of these linear stabilizers may deteriorate, especially when compared with that of stabilizers designed using modern control techniques. A Neural PSS, trained with a set of local linear controllers, is applied to establish the regions where a Conventional PSS shows low performance. Using non-linear digital simulations of a synchronous machine connected to an infinite-bus system and a multi-machine power system the Neural PSS is assessed showing superiority in those regions.
\end{abstract}

KEYWORDS: Power System Stabilizers, Neural Networks, Power System Control, Dynamic Stability, Excitation Con-

\footnotetext{
Artigo Submetido em 08/05/2003

1a. Revisão em 12/09/2005

2a. Revisão em 31/05/2006

Aceito sob recomendação do Editor Associado

Prof. Glauco Nery Taranto
}

trol.

\section{RESUMO}

A utilização de Estabilizadores de Sistemas de Potência (ESP), para amortecer oscilações eletromecânicas de pequena magnitude e baixa freqüência, é cada vez mais importante na operação dos modernos sistemas elétricos. Estabilizadores convencionais, com estrutura e parâmetros fixos, têm sido utilizados com essa finalidade há algumas décadas, porém existem regiões de operação do sistema nas quais esses estabilizadores lineares não são tão eficientes, especialmente quando comparados com estabilizadores projetados através de modernas técnicas de controle. Um ESP Neural, treinado a partir de um conjunto de controladores lineares locais, é utilizado para investigar em quais regiões de operação do sistema elétrico o desempenho do estabilizador a parâmetros fixos é deteriorada. O melhor desempenho do ESP Neural nessas regiões de operação, quando comparado com o ESP convencional, é demonstrado através de simulações digitais não-lineares de um sistema do tipo máquina síncrona conectada a um barramento infinito e de um sistema com quatro geradores. 
PALAVRAS-CHAVE: Estabilizadores de Sistemas de Potência, Redes Neurais, Controle de Sistemas de Potência, Estabilidade Dinâmica, Controle de Excitação.

\section{INTRODUÇÃO}

Uma das características mais comuns dos atuais sistemas elétricos de potência é o funcionamento de forma interligada, conectando grandes unidades geradoras, próximas de seus limites de capacidade, através de longas linhas de transmissão. A interligação de sistemas de potência justifica-se por motivos econômicos e de segurança, pois permite que a operação do sistema elétrico seja bastante flexível. Entretanto, do ponto de vista das margens de estabilidade, operar o sistema desta forma faz com que a capacidade de amortecer as oscilações eletromecânicas de baixa frequência, intrínsecas ao sistema de potência, possa ser drasticamente reduzida (Rogers, 2000). Este problema torna-se ainda mais grave quando há a presença de Reguladores Automáticos de Tensão (RAT), que utilizam eletrônica de potência nos geradores do sistema, com ganho elevado e resposta rápida (Acha et al., 2002; Mohan et al., 2003). Esse tipo de RAT, embora excelente para melhorar a estabilidade transitória e o controle eficiente da tensão, contribui para reduzir as margens de estabilidade dinâmica dos sistemas elétricos.

A solução geralmente adotada para amortecer essas oscilações indesejáveis de baixa frequiência é a utilização dos chamados Estabilizadores de Sistemas de Potência (ESPs), que fornecem um sinal estabilizante auxiliar a ser injetado na malha de regulação de tensão dos geradores (Kundur, 1994). O ESP fornece uma componente de torque elétrico de amortecimento (em fase com os desvios de velocidade do rotor do gerador), o que ajuda no amortecimento das oscilações do sistema.

Os estabilizadores convencionais, denominados neste trabalho de ESPCs, possuem estrutura e parâmetros fixos. Desta forma, o seu projeto é feito através de técnicas de controle clássico, com o sistema de potência sendo representado através de um sistema linearizado em torno de um ponto de operação específico. Embora sintonizados para um determinado ponto de operação, os estabilizadores convencionais geralmente apresentam um bom desempenho para uma grande faixa de condições de operação do sistema elétrico (Larsen e Swan, 1981). Porém, é importante destacar que se outros ESPCs fossem projetados para cada ponto de operação, os resultados poderiam ser ainda melhores.

Nos últimos anos, vários métodos têm sido propostos para obter estabilizadores que apresentem um desempenho melhor que o apresentado pelo ESPC. Dentre esses métodos, pode-se citar: Ganhos Programados, Controle Adaptativo, Redes Neurais e Sistemas Lógicos Fuzzy (Pierre, 1987; Gu e Bollinger, 1989; Hsu e Chen, 1991; Hiyama et al., 1996; Hunt e Johansen, 1997; Shamsollahi e Malik, 1999). É uma característica comum a todos esses métodos, apresentar um desempenho bastante similar ao estabilizador convencional em condições de operação ao redor do ponto de operação utilizado para sintonizar o ESPC. Porém, quanto mais o ponto de operação se afasta da condição utilizada para projetar o ESPC, os estabilizadores projetados por essas outras técnicas de controle costumam apresentar um desempenho muito superior ao obtido com o estabilizador convencional.

O objetivo principal deste artigo é investigar, de forma qualitativa, em que regiões de operação do sistema esta última situação ocorre. O conjunto de parâmetros de um ESP a ganhos programados (Barreiros et al., 1999) é utilizado para treinar uma Rede Neural Artificial (RNA), através do conhecido algoritmo de retropropagação do erro. Devido à capacidade da RNA de fazer o mapeamento de qualquer função não-linear com a precisão desejada (Haykin, 1998), é possível observar as variações que ocorrem nos parâmetros do controlador com a mudança no ponto de operação do sistema de potência.

Neste trabalho será apresentado um estudo detalhado para a utilização do ESP Neural proposto para o caso clássico de um sistema máquina síncrona - barramento infinito. Além disto, a aplicação do método de controle proposto em um sistema multimáquinas também é investigada, demonstrando-se assim a viabilidade da utilização da estratégia proposta também em sistemas interligados.

\section{ESP A GANHOS PROGRAMADOS}

Um controlador a ganhos programados é, basicamente, um conjunto de controladores lineares, onde cada um desses controladores é projetado para um ponto de operação específico. Desta forma, quando o sistema encontra-se em determinada condição de operação, o sinal de controle é gerado pelo controlador previamente projetado para essa condição atual de operação. Neste trabalho, um ESP a ganhos programados (ESPGP) é utilizado para fornecer os parâmetros do controlador em vários pontos de operação do sistema, fornecendo assim os padrões de treinamento para uma RNA.

Para o projeto do ESPGP, que será utilizado no sistema máquina síncrona - barramento infinito, a região de operação da máquina síncrona no plano $P$ x $Q$ (das potências elétrica ativa e reativa), foi dividida em cerca de 100 regiões, consideradas como sendo suficientes para representar adequadamente as condições de operação do sistema de potência. Em cada uma dessas regiões, um controlador linear foi obtido assumindose que o ponto de operação é representado pelo valor central de $P$ e $Q$ naquela região específica, como mostra a Figura 1. 


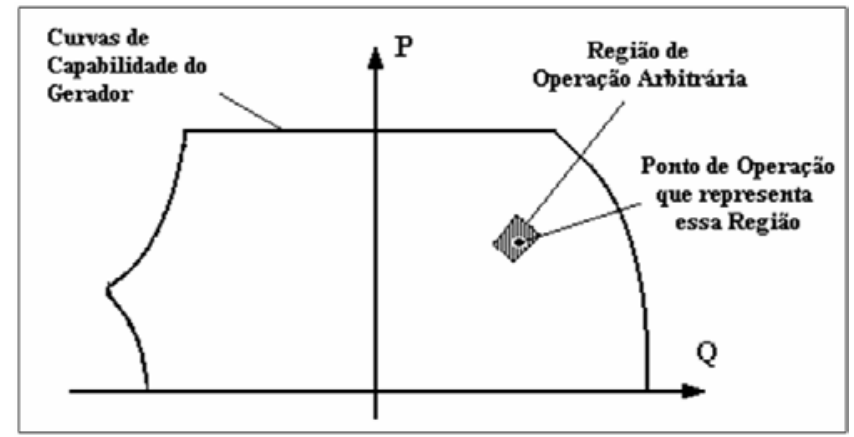

Figura 1: Regiões do plano PxQ usadas no projeto do ESP

Estas regiões são limitadas pelas curvas de capabilidade do gerador (Guru e Hiziroglu, 2001), tendo a forma de setores, divididos com a precisão desejada. A quantidade de setores foi escolhida de tal forma que pudessem representar bem as regiões em que a presença do ESP é mais necessária, além de serem capazes de evitar mudanças muito bruscas no sinal de controle, quando o ponto de operação varia de acordo com as condições de operação do sistema (Hunt e Johansen, 1997; Barreiros et al., 1999). Em cada uma destas regiões, obtevese um modelo discreto linear da planta na forma:

$$
\begin{gathered}
y(k T)=b_{0} u(k T-T)+b_{1} u(k T-2 T)+ \\
b_{2} u(k T-3 T)-a_{1} y(k T-T)- \\
a_{2} y(k T-2 T)-a_{3} y(k T-3 T)
\end{gathered}
$$

onde $\mathrm{T}$ é o período de amostragem do sistema, $k T$ representa o instante de tempo discreto atual, $u$ e $y$ são a entrada e saída da planta, respectivamente. Em todas as simulações realizadas neste trabalho, adotou-se um valor de $T=100 \mathrm{~ms}$.

Os parâmetros $a_{i}$ e $b_{i}$ da equação (1) são estimados através do método dos Mínimos Quadrados Recursivos (MQR) (Landau, 1990). A partir do modelo linear da planta, um controlador é projetado através de uma técnica de alocação de pólos, que consiste em deslocar radialmente na direção da origem do plano-z os pólos com baixo amortecimento (Cheng et al., 1986). Assim, os pólos desejados para o sistema em malha fechada podem ser obtidos multiplicando-se as magnitudes dos pólos originais (mal amortecidos) por uma constante real $\alpha<1$. Caso existam pólos instáveis, estes são previamente substituídos por seus recíprocos. Desta forma, os parâmetros do controlador assim projetado podem ser representados pela seguinte equação de diferença:

$$
\begin{aligned}
& u(k T)=g_{0} y(k T)+g_{1} y(k T-T)+ \\
& g_{2} y(k T-2 T)-h_{1} u(k T-T)-h_{2} u(k T-2 T)
\end{aligned}
$$

Os parâmetros do controlador $g_{i}$ e $h_{i}$ podem ser determinados através da resolução do seguinte sistema linear, obtido a partir da equação diofantina presente na técnica de alocação de pólos (Aström e Wittenmark, 1997):

$$
\left[\begin{array}{ccccc}
1 & 0 & b_{0} & 0 & 0 \\
a_{1} & 1 & b_{1} & b_{0} & 0 \\
a_{2} & a_{1} & b_{2} & b_{1} & b_{0} \\
a_{3} & a_{2} & 0 & b_{2} & b_{1} \\
0 & a_{3} & 0 & 0 & b_{2}
\end{array}\right]\left[\begin{array}{c}
h_{1} \\
h_{2} \\
g_{0} \\
g_{1} \\
g_{2}
\end{array}\right]=\left[\begin{array}{c}
(\alpha-1) a_{1} \\
\left(\alpha^{2}-1\right) a_{2} \\
\left(\alpha^{3}-1\right) a_{3} \\
0 \\
0
\end{array}\right]
$$

O parâmetro $\alpha$ deve ser escolhido na faixa entre 0 e 1 e, nesta aplicação, que utiliza um intervalo de amostragem de $100 \mathrm{~ms}$, adotou-se $\alpha=0,75$. Este valor permite um bom amortecimento dos pólos dominantes do sistema de potência, sem excitar demasiadamente os modos de freqüência mais elevada (Silva e Barreiros, 1992).

Este procedimento é repetido para cada um dos pontos de operação do sistema mencionados anteriormente, resultando em um conjunto também com cerca de 100 controladores. Se o ESPGP estivesse atuando no sistema, ao receber os valores que definem o ponto de operação atual, escolheria dentre os controladores disponíveis qual o que deveria gerar o sinal de controle. É esse conjunto com os parâmetros do controlador que serve como padrão de treinamento para um ESP Neural, como será mostrado a seguir.

\section{ESP NEURAL}

Apesar do número considerável de pontos de operação utilizados pelo ESPGP, em determinadas situações existem algumas diferenças, com relação aos parâmetros do controlador, entre o ponto de operação dentro de uma região e o ponto central que foi escolhido para representar esta mesma região. Por esta razão, uma RNA do tipo perceptron multicamadas foi treinada, através do algoritmo de retropropagação do erro, para funcionar como um supervisor e interpolador, fornecendo os parâmetros do controlador mesmo para pontos de operação que não foram utilizados durante o processo de treinamento. A duração do treinamento foi de 500.000 épocas, utilizando uma taxa de aprendizado de 0,2 e um fator de momento igual a 0,9 .

O Estabilizador de Sistemas de Potência Neural (ESPN) é uma RNA estática (seus pesos não são alterados após o término do processo de treinamento), cujas entradas são os valores atuais de potência ativa e reativa fornecidos pela máquina síncrona. A arquitetura da rede neural, mostrada na Figura 2, é composta por 2 camadas escondidas com 10 e 7 neurônios, respectivamente, e com função de ativação do tipo sigmóide (Haykin, 1998). Esta arquitetura foi escolhida através de tentativa e erro, sendo a que apresentou melhor resultado dentre as várias arquiteturas testadas. Para fornecer os parâmetros do controlador, a camada de saída é formada 


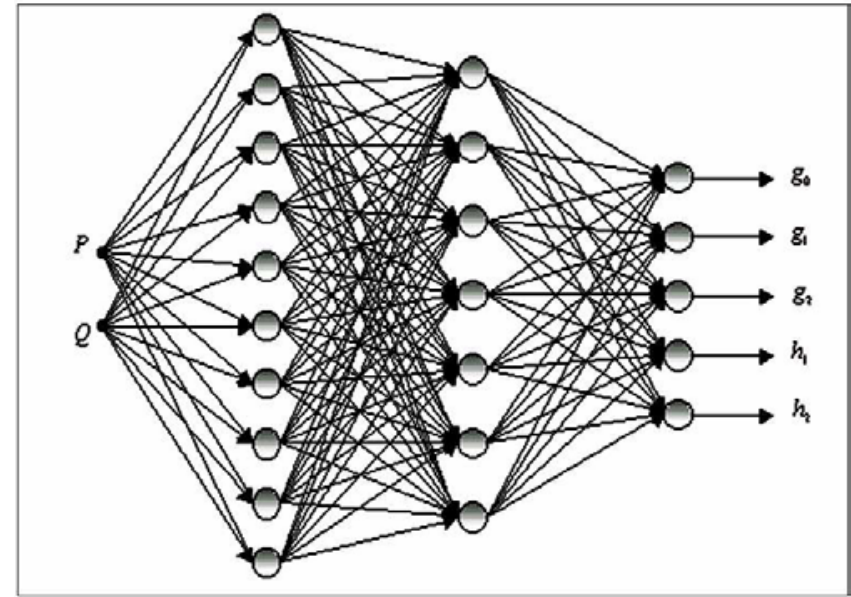

Figura 2: Arquitetura da RNA utilizada pelo ESP Neural

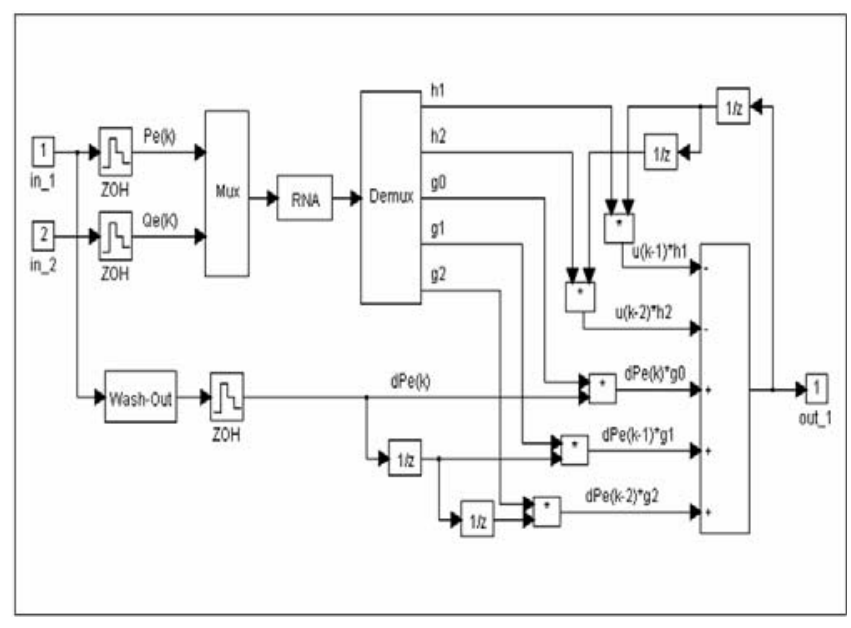

Figura 3: Estrutura do ESP Neural

por 5 neurônios com função de ativação linear. A estrutura completa do ESP Neural, que usa sinal de potência num esquema de realimentação negativa, é mostrada na Figura 3.

Com o ESP Neural já treinado, aplicou-se em suas entradas valores de potência ativa e reativa cobrindo uma ampla faixa de valores e observou-se qual o comportamento dos parâmetros do controlador nessas condições de operação. Os valores de potência ativa aplicados foram entre 0 e 1 pu, enquanto a potência reativa variou entre -1 e $1 \mathrm{pu}$, resultando em um conjunto de 2500 controladores.

É importante notar que vários dos pontos de operação considerados não são condições de operação válidas, ou seja, são situações que nunca irão ocorrer na operação viável de um sistema de potência real. Porém, esses pontos foram mantidos e são mostrados nas Figuras 4 a 8 para mostrar como os parâmetros dos controladores aqui projetados variam com a

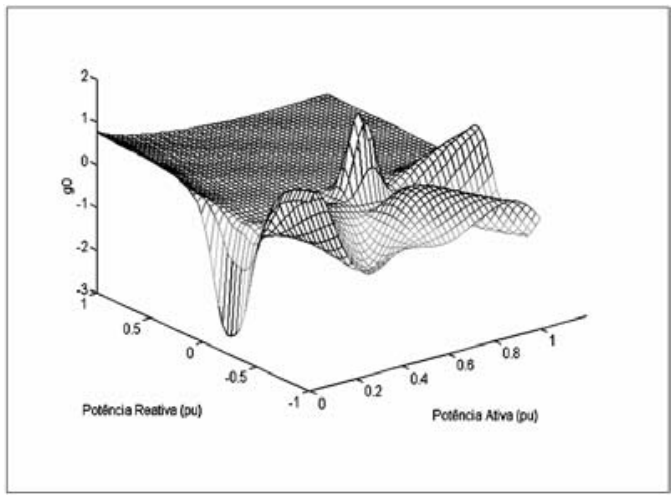

Figura 4: Parâmetro $g_{0}$ do controlador

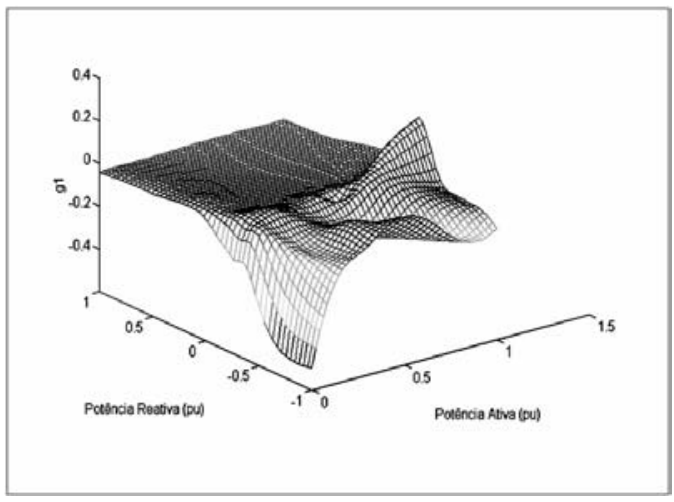

Figura 5: Parâmetro $g_{1}$ do controlador

mudança da condição de operação do sistema.

Os resultados fornecidos pelo ESP Neural mostram que os parâmetros do controlador não sofrem grandes modificações quando a máquina síncrona está fornecendo potência reativa ao sistema. Porém, quando a potência reativa é negativa (o gerador está absorvendo reativo), os parâmetros do controlador sofrem variações muito bruscas, mesmo para condições de operação bastante próximas. Esta é a principal característica do ESP Neural aplicado ao sistema máquina síncrona barramento infinito.

Como os estabilizadores convencionais geralmente são sintonizados em uma região de $Q$ positivo (Larsen e Swan, 1981), isto pode explicar a razão desses estabilizadores apresentarem um desempenho aceitável em todas as regiões onde o gerador fornece reativo ao sistema. Entretanto, quando o sistema está trabalhando em uma região com $Q$ negativo, o desempenho de um ESPC ao ser comparado com outros métodos de projeto (como controle adaptativo, redes neurais ou lógica fuzzy) é quase sempre bastante inferior, com o ESPC não sendo capaz de amortecer tão rapidamente as oscilações eletromecânicas do sistema. 


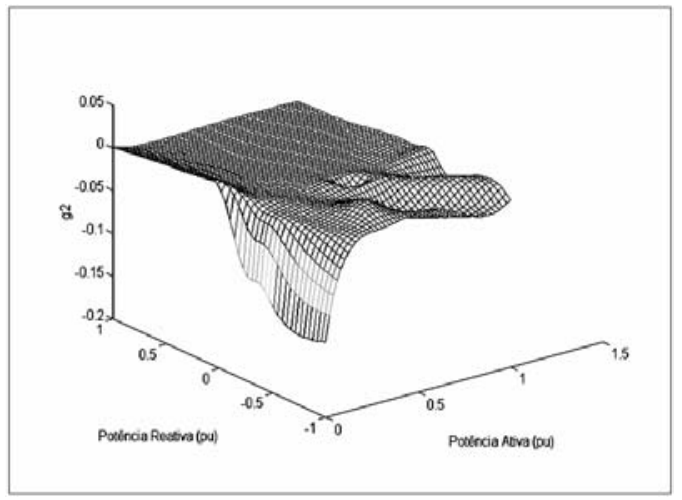

Figura 6: Parâmetro $g_{2}$ do controlador

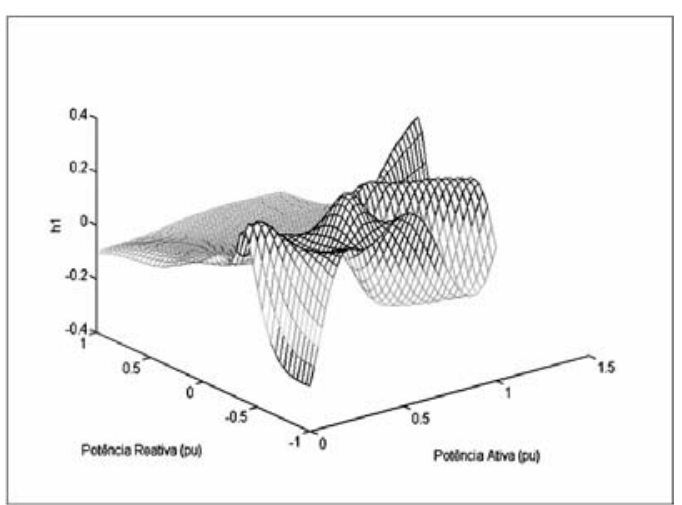

Figura 7: Parâmetro $h_{1}$ do controlador

\section{RESULTADOS DE SIMULAÇÕES: CASO MÁQUINA SÍNCRONA - BARRAMENTO INFINITO}

Um sistema do tipo máquina síncrona - barramento infinito foi utilizado para realizar os testes do ESP Neural proposto. Neste sistema, o gerador é conectado a um sistema do tipo barra infinita (representado por um único barramento com tensão e freqüência constantes) através de uma linha de transmissão equivalente de um circuito duplo $\left(R_{e}+j X_{e}\right)$. O modelo utilizado para o gerador é não-linear de $5^{a}$ ordem, representando um gerador de pólos salientes com enrolamentos amortecedores (Ong, 1998; Arrilaga, 1983) e cujos parâmetros são mostrados no Apêndice. O sistema de excitação utilizado é representado por um sistema de $1^{a}$ ordem, com ganho elevado e constante de tempo rápida, além de limitadores $\left(K_{a}=200, T_{a}=0,03\right.$ s e $\left.E f_{\text {lim }}= \pm 6 \mathrm{pu}\right)$.

Um dos controladores do ESPGP foi escolhido para ser o ESPC que será utilizado comparativamente nas simulações para avaliar o desempenho do ESP Neural. A escolha do ponto de operação para o ESPC foi feita com base nas recomendações tradicionais para o projeto de um estabilizador

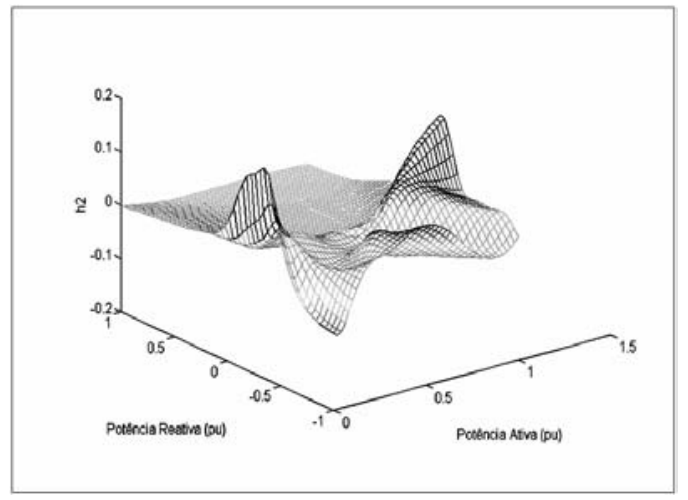

Figura 8: Parâmetro $h_{2}$ do controlador

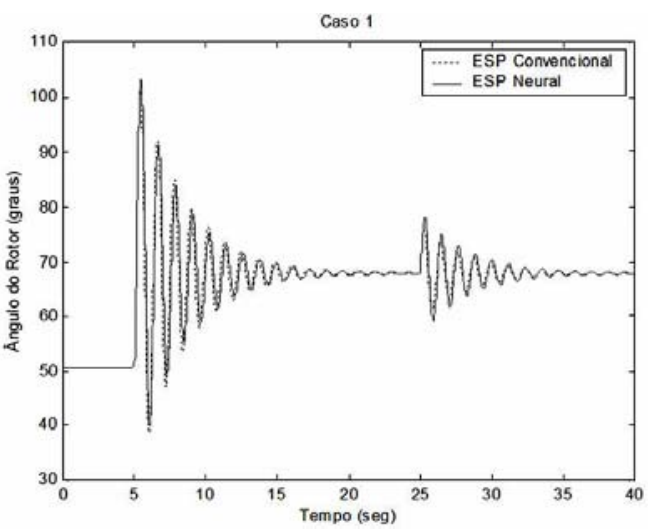

Figura 9: Ângulo do rotor para o Caso 1

com estrutura e parâmetros fixos (Larsen e Swan, 1981) e, na saída de todos os estabilizadores, limita-se o sinal de controle em $\pm 0,1 \mathrm{pu}$. Diversas simulações foram efetuadas e os resultados de 2 casos representativos são apresentados a seguir.

Caso 1 - O gerador está operando com $P=0,75$ pu e $Q=0,0998 \mathrm{pu}$. Um curto-circuito trifásico é aplicado na saída da máquina síncrona em $\mathrm{t}=5 \mathrm{~s}$, com duração de $0,1 \mathrm{~s}$ e perda de uma das linhas de transmissão do circuito duplo. Após esta falta, o novo ponto de operação resultante da mudança estrutural no sistema ( $P=0,75$ pu e $Q=0,2220 \mathrm{pu})$ é mais crítico que o ponto de operação inicial, já que o ângulo do rotor passa de uma condição em torno de $50^{\circ}$ para um valor maior que $65^{\circ}$. Em $\mathrm{t}=25 \mathrm{~s}$, um novo curto-circuito ocorre no mesmo ponto, desta vez com duração de $0,025 \mathrm{~s} \mathrm{e}$ sem perda de linha.

Quando não há um estabilizador no sistema, a estabilidade angular é perdida logo após a primeira falta e, por isso, seu gráfico não é mostrado. A evolução do ângulo do rotor do gerador é mostrada na Figura 9 para os casos com o ESPC e o ESPN. 


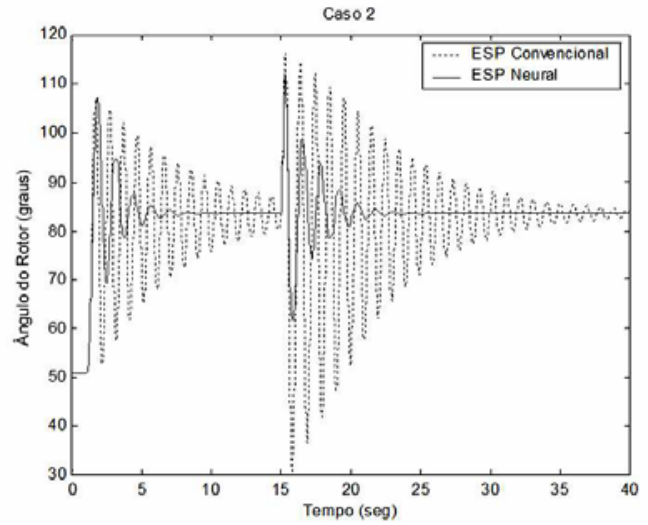

Figura 10: Ângulo do rotor para o Caso 2

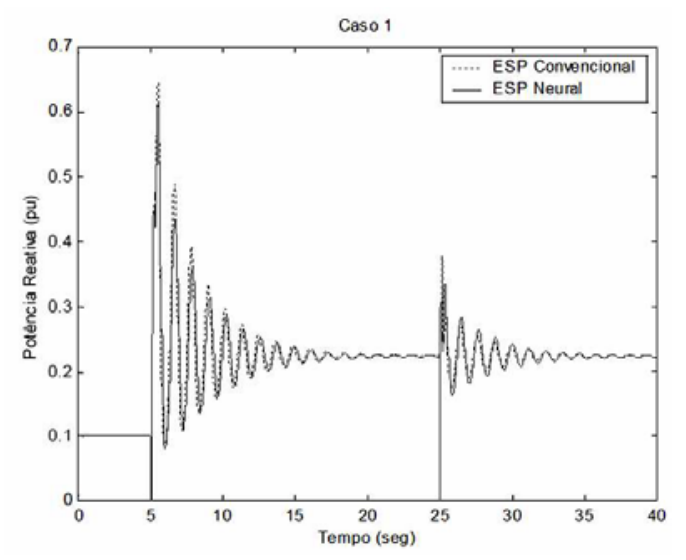

Figura 11: Potência Reativa para o Caso 1

Para este caso em particular, tanto o ESPC quanto o ESPN apresentam um desempenho bastante satisfatório, amortecendo rapidamente as oscilações que surgem no sistema. Isto pode ser explicado porque a condição de operação inicial está próxima da condição de operação utilizada para sintonizar o ESPC. Um outro ponto importante, é o fato que em quase nenhum momento durante a simulação a potência reativa tornou-se negativa, fazendo com que os parâmetros do ESPN não apresentassem variações consideráveis quando comparados com os parâmetros do ESPC.

Caso 2 - O sistema opera na mesma condição inicial do Caso 1 e aplica-se uma redução de $15 \%$ na tensão de referência do sistema de excitação em $\mathrm{t}=1 \mathrm{~s}$. Como consequiência desta primeira falta, o sistema passa a operar na região de $Q$ negativo. Em seguida, em $\mathrm{t}=15 \mathrm{~s}$, um curto-circuito trifásico no terminal do gerador é aplicado, com duração de 0,1 s e sem perda de linha de transmissão. A resposta do ângulo do rotor para o ESPC e para o ESPN é mostrada na Figura 10, onde se percebe facilmente a superioridade do segundo estabilizador.

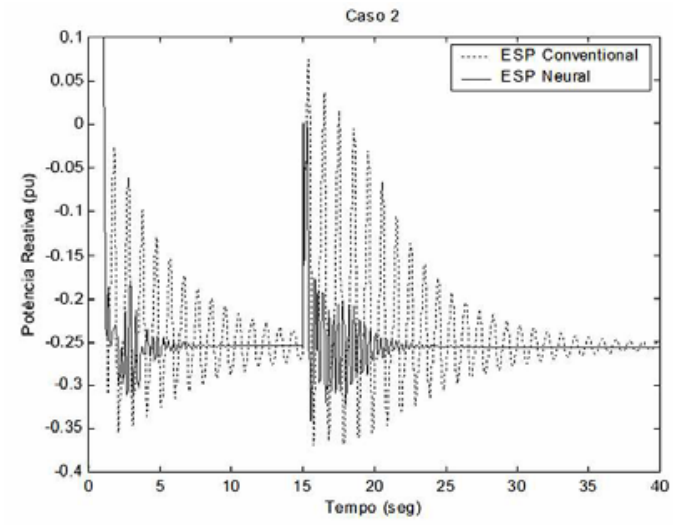

Figura 12: Potência Reativa para o Caso 2

Embora o sistema continue estável com os dois tipos de estabilizador, como o ESPC foi sintonizado para uma condição de operação bastante diferente daquela em que o sistema está operando, seu desempenho é extremamente inferior ao obtido com o ESP Neural. Nas Figuras 11 e 12 são mostradas as curvas de potência reativa para os Casos 1 e 2, respectivamente, onde é possível se observar que no primeiro caso simulado a potência reativa é predominantemente positiva, enquanto que no segundo caso, durante a maior parte do tempo de simulação, a máquina está operando absorvendo reativo do sistema.

Para ilustrar melhor o comportamento do sistema nos dois casos simulados, apresenta-se a seguir, para ambos os casos, gráficos do tipo plano de fase entre o ângulo do rotor e sua velocidade (Figuras 13 e 14), e entre as potências reativa e ativa (Figuras 15 e 16). Observando-se estes gráficos, fica bastante claro perceber o desempenho superior do ESP Neural no segundo caso, quando o sistema opera com um valor predominantemente negativo de potência reativa. Mostrouse anteriormente nas Figuras 4 a 8 que os parâmetros do controlador variam bastante quando $Q$ é negativo, porém muito pouco nos casos de $Q$ positivo. Isto também pode ser comprovado nas Figuras 17 a 21, que mostram a evolução ao longo do tempo dos parâmetros do ESP Neural com a mudança nas condições de operação do sistema para os dois casos simulados.

\section{RESULTADOS DE SIMULAÇÕES: CASO MULTIMÁQUINAS}

$\mathrm{Na}$ seção anterior foram apresentadas as propriedades e os resultados de simulação obtidos na avaliação do desempenho da estrégia de controle baseada em ESP neural aplicado a um sistema do tipo máquina síncrona - barramento infinito. Embora este sistema seja relativamente simples, com o mesmo é possível que o projetista possa obter uma avaliação 


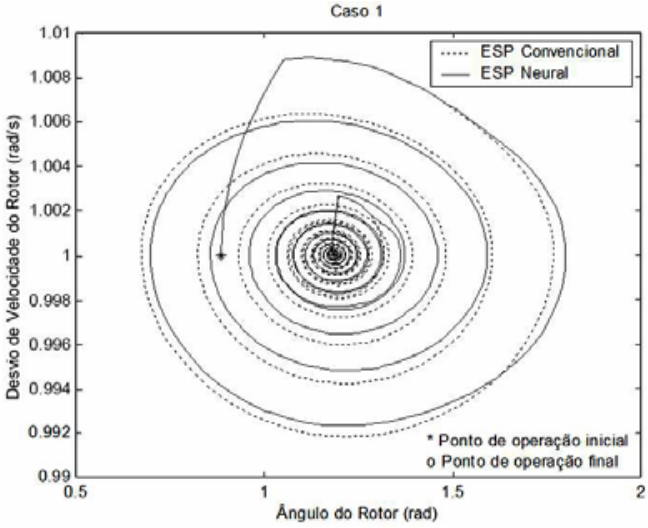

Figura 13: Plano de Fase $\delta$ x $\omega$ para o Caso 1

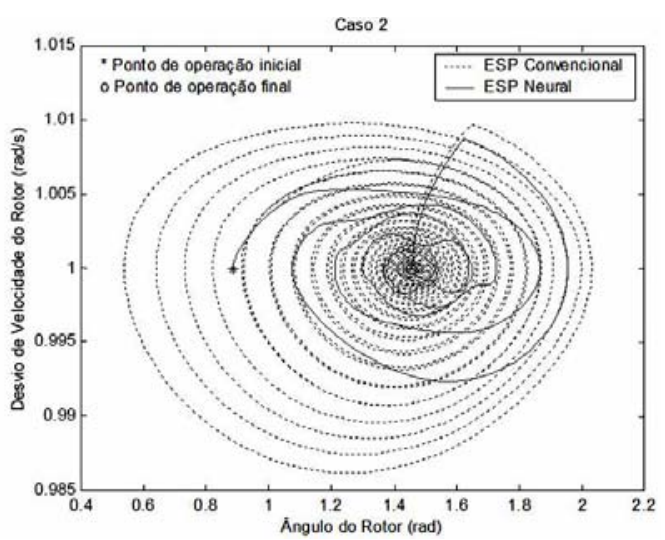

Figura 14: Plano de Fase $\delta$ x $\omega$ para o Caso 2

qualitativa inicial referente à sensibilidade dos parâmetros de projeto da estratégia de controle neural sendo testada, permitindo assim uma melhor seleção dos parâmetros de projeto.

No entanto, para que se possa indicar a estratégia de controle baseada no ESP neural como candidata à utilização em sistemas interligados de grande porte, é necessário também avaliar o desempenho da estratégia quando esta é aplicada em sistemas multimáquinas, de modo a investigar a existência de eventuais efeitos colaterais resultantes de interações entre o ESP neural e os ESPs convencionais instalados nos diversos geradores instalados no sistema interligado.

\subsection{Descrição do Sistema Multimáquinas}

O desempenho do ESP neural, proposto neste trabalho, foi investigado em um modelo de sistema multimáquinas simples (Figura 22), composto de quatro geradores iguais, de potência nominal 900 MVA cada um, os quais são dispostos de modo a formar um sistema de potência interligado contendo duas áreas de geração. Este sistema multimáquinas,

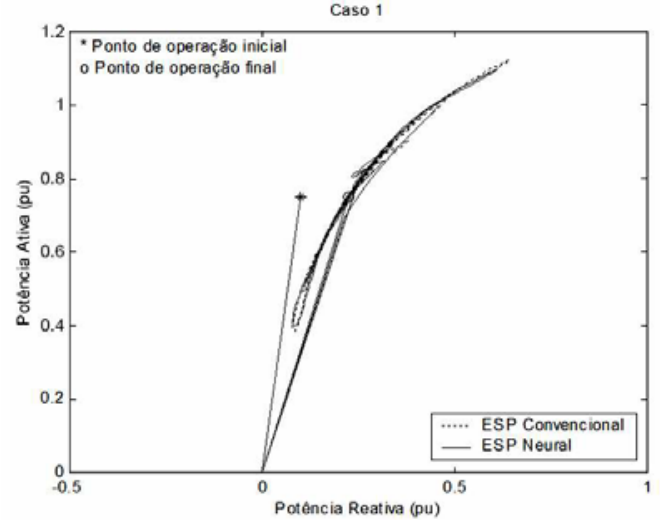

Figura 15: Gráfico de $Q \times P$ para o Caso 1

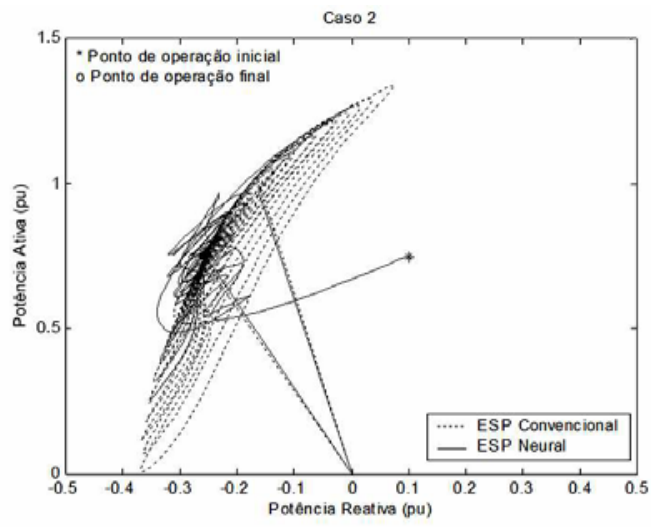

Figura 16: Gráfico de $Q$ × $P$ para o Caso 2

embora seja simples, exibe uma série de fenômenos que normalmente são observados em sistemas de grande porte. Os parâmetros das máquinas, dos controladores e da rede elétrica, para este sistema, podem ser encontrados em Kundur (1994).

Os sistemas de excitação dos geradores são todos do tipo estático de ação rápida, e equipados com reguladores automáticos de tensão de ganho elevado $\left(K_{a}=200\right)$. No caso base, o sistema multimáquinas estará operando de modo que a área de geração 1 estará exportando $400 \mathrm{MW}$, para a área 2, através de uma longa linha de interligação. O sistema multimáquinas exibe dois modos locais de oscilação eletromecânica (um em cada área de geração) e um modo interárea.

Na condição operacional base cada gerador fornecerá uma potência ativa de aproximadamente $700 \mathrm{MW}$, com o modo interárea sendo instável, apresentando amortecimento negativo $(f=0,61 \mathrm{~Hz}$ e $\zeta=-0,008)$. Para esta condição de operação, cada um dos modos locais apresenta uma frequiência de oscilação de aproximadamente $1 \mathrm{~Hz}$ e, embora sejam estáveis nesta condiçao operacional, apresentam um baixo 


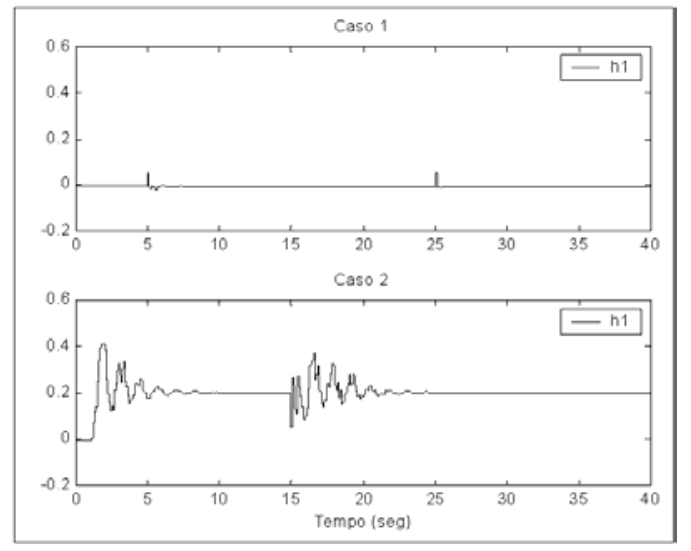

Figura 17: Parâmetro $h_{1}$ do ESP neural para os Casos 1 e 2

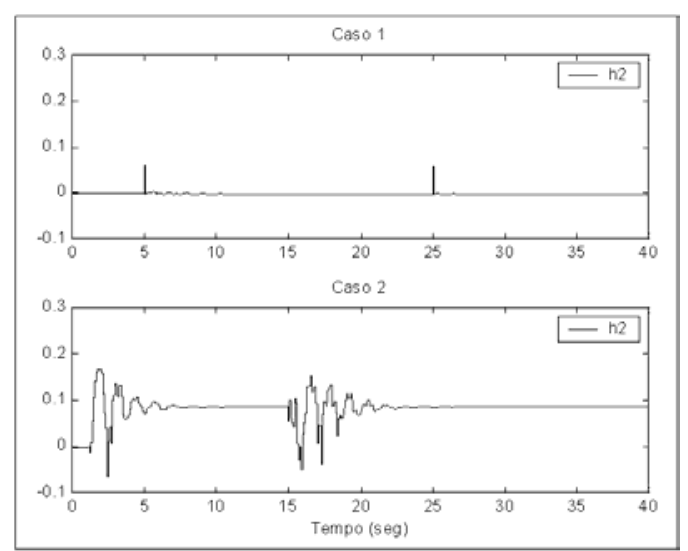

Figura 18: Parâmetro $h_{2}$ do ESP neural para os Casos 1 e 2

\subsection{Identificação dos Modelos Locais}

Para se verificar o desempenho do método proposto neste trabalho, foi projetado um ESP neural para substituir o ESP convencional do gerador 1 . O desempenho quando se utiliza o ESP neural será comparado com o obtido pelo ESP convencional em G1, sendo que em ambos os casos serão mantidos em operação os ESPs convencionais nos geradores da área 2. A opção de não se utilizar ESP em G2 durante as simulações é apenas para evidenciar que o amortecimento do modo local entre os geradores G1 e G2 deve-se primordialmente à ação do ESPN projetado.

Primeiramente, com os geradores da área 1 operando sem usar ESP, foi identificado um conjunto de modelos ARX representivos de diversos pontos de operação obtidos para diferentes níveis de carregamento do gerador e de fluxo de potência na linha de interligaçao 7-8 (Tabela 1), utilizando o método dos mínimos quadrados. Foram efetuados estudos de autocorrelação do resíduo onde foi observado que o uso 


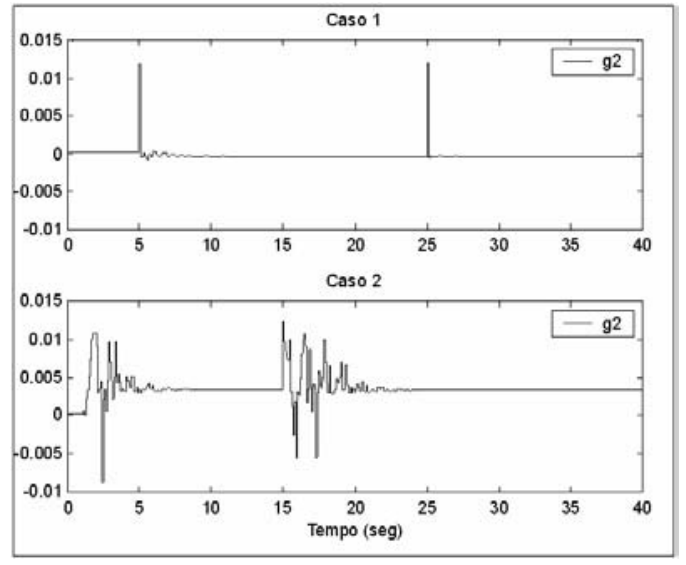

Figura 21: Parâmetro $g_{2}$ do ESP neural para os Casos 1 e 2

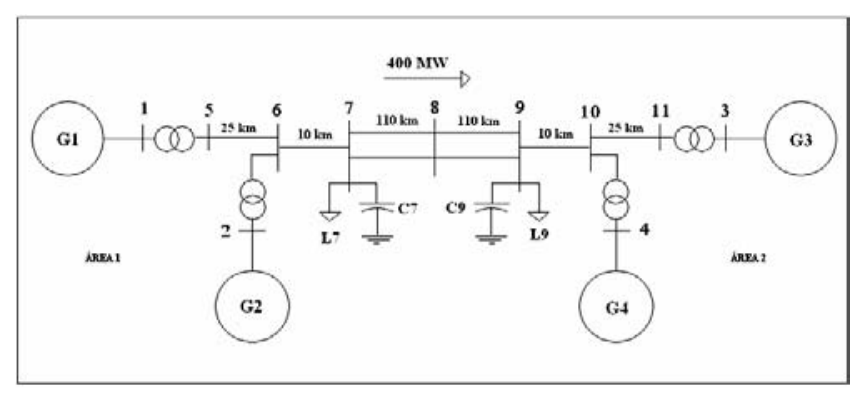

Figura 22: Sistema de teste com duas áreas de geração.

de modelos ARX de $5^{a}$ ordem, com atraso de um intervalo de amostragem, apresenta um bom desempenho na representação da dinâmica da planta, para os pontos de operação considerados, capturando eficientemente a informaçao sobre o modo menos amortecido de oscilação eletromecânica observável no sinal de potência ativa nos terminais do gerador G1.

Juntamente com a potência ativa nos terminais do gerador $1, \mathrm{P}_{G 1}$, a potência de interligação $\mathrm{P}_{78}$ foi também selecionada como variável de operação neste estudo de simulação, sendo utilizada como um dos sinais de entrada do controlador neural. Isso se deve ao fato que o carregamento da linha de interligação poderá afetar o modos eletromecânicos deste sistema. No entanto, para o caso de outros sistemas multimáquinas, a escolha das variáveis de operação mais adequadas dependerá da configuração do sistema particular em estudo.

\subsection{Projeto dos Controladores Locais}

Os parâmetros dos modelos locais foram então utilizados no projeto de um conjunto de controladores locais, através do uso da técnica de posicionamento de pólos. Foi utilizado o método de deslocamento radial dos pólos em direção ao centro do circulo unitário. O intervalo de amostragem utilizado

\begin{tabular}{|l|l|c|c|}
\hline & & \multicolumn{2}{|c|}{$\begin{array}{c}\text { Pontos de operação } \\
\text { (em pu, base100MVA) }\end{array}$} \\
\hline & & PG1 & P78 \\
\hline \multirow{4}{*}{ Fluxo 7-8 } & CASO BASE & 7.00 & 4.0053 \\
\cline { 2 - 4 } & CASO \#1 & 1.00 & 0.3235 \\
\cline { 2 - 4 } & CASO \#2 & 2.00 & 0.6049 \\
\cline { 2 - 4 } & CASO \#3 & 3.00 & 0.7736 \\
\cline { 2 - 4 } & CASO \#4 & 4.00 & 1.6287 \\
\cline { 2 - 4 } & CASO \#5 & 5.00 & 2.5729 \\
\cline { 2 - 4 } & CASO \#6 & 6.00 & 3.0996 \\
\hline \multirow{4}{*}{ Fluxo 8-7 } & CASO \#7 & 1.00 & -0.6765 \\
\cline { 2 - 4 } & CASO \#8 & 4.00 & -1.7722 \\
\cline { 2 - 4 } & CASO \#9 & 6.00 & -3.0030 \\
\hline
\end{tabular}

Tabela 1: Pontos de operaçao utilizados na identicaçao de modelos ARX e no projeto de controladores locais.

foi de $100 \mathrm{~ms}$. O objetivo do projeto dos controladores locais foi, neste caso, o de ampliar o amortecimento do modo subamortecido observado pelo modelo, para o valor de $\zeta=0.5$, isso sem afetar substancialmente o valor da freqüência natural de oscilação do referido modo.

\subsection{Treinamento do ESP Neural}

Os valores dos parâmetros dos controladores locais projetados foram utilizados como padrões para o treinamento de uma rede neural do tipo percetron multicamadas, cujas saídas serão cada um dos parâmetros do controlador $\left(\mathrm{g}_{0}, \mathrm{~g}_{1}, \mathrm{~g}_{2}, \mathrm{~g}_{3}, \mathrm{~g}_{4}, \mathrm{e} \mathrm{h}_{1}, \mathrm{~h}_{2}, \mathrm{~h}_{3}, \mathrm{~h}_{4}\right)$. A RNA, após o processo de treinamento, é utilizada em tempo real para fornecer (interpolar) os valores dos parâmetros do ESP neural $\left(\mathrm{g}_{0}\left(\mathrm{P}_{1}, \mathrm{P}_{78}\right), \ldots, \mathrm{h}_{4}\left(\mathrm{P}_{1}, \mathrm{P}_{78}\right)\right)$, para cada valor medido do par de variáveis de operação $\left(\mathrm{P}_{1}, \mathrm{P}_{78}\right)$. Os parâmetros selecionados para o treinamento das redes neurais foram os seguintes:

Estrutura: dois parâmetros de entrada (PG1 e P78), duas camadas ocultas com 20 neurônios cada com funções de ativaçao do tipo sigmóide (o número de neurônios das camadas ocultas foi selecionado por tentativa e erro) e nove neurônios na camada de saída com função de ativação linear (conjunto de parâmetros do controlador).

Aprendizado: para o treinamento dos pesos também foi utilizado o método de aprendizado por retro-propagação do erro, com uma taxa de aprendizado de valor igual a 0,2 e um fator de momento de valor igual a 0,9 . Utilizou-se 500 mil épocas para o treinamento da rede, avaliando-se que o erro quadrático da rede ao final desse período era aceitável. 


\subsection{Avaliação de Desempenho do ESP Neural}

Após finalizado o treinamento do ESP neural, o mesmo foi implantado no sistema de excitaçao do gerador G1 e seu desempenho no auxílio ao amortecimento do modo de oscilaçao observável através do sinal $\mathrm{P}_{G 1}$. O desempenho do ESP neural é comparado ao desempenho obtido quanto se utiliza um ESP convencional em G1.

\subsubsection{Desempenho para o Caso Base}

Na figura 23 é apresentado a resposta dos ângulos dos rotores das máquinas G2 (na área 1) e de G3 (na área 2), em relação ao ângulo do rotor de G1, $\delta_{12}$ e $\delta_{13}$, respectivamente, para o sistema operando na condição operacional base descrito em (Kundur, 1994) (ver Tabela 1), com um fluxo de 400 MW no sentido da área 1 para a área 2 . O sistema inicia operando em regime permanente e, em $t=1,0 \mathrm{~s}$, sofre uma falta do tipo curto-circuito trifásico no barramento 8, com uma duração de $100 \mathrm{~ms}$, sem perda de linha de transmissão após sanado o curto-circuito.

Pode-se observar que, após o curto-circuito, o desempenho dinâmico do sistema equipado com ESPs convencionais apresenta um excelente desempenho no ponto de operaçao base, amortecendo eficientemente os modos locais e interárea, em aproximadamente 5 segundos após o curto- circuito (curva em linha tracejada, na figura 23). Este resultado era de se esperar, já que os ESPs convencionais foram efetivamente projetados para o ponto de operação base. O desempenho do sistema com um ESP neural em G1, é mostrado também na figura 23 (curva em linha cheia). Pode-se observar que o desempenho do sistema quando se utiliza um ESP neural em G1, é também satisfatório, contribuindo para amortecer as oscilaçoes em intervalo de tempo da ordem de 6 segundos e sem apresentar uma interferência adversa observável em relação à interaçao com os demais controladores do sistema multimáquinas. Observa-se, ainda, que o esforço de controle do ESP neural, para este caso, foi apenas ligeiramente maior do que o esforço de controle obtido quando se utiliza um ESP convencional em G1.

\subsubsection{Desempenho com a Inversão no Fluxo de Potência entre as Áreas 1 e 2}

Para verificar a robustez do controlador neural, em relação à sua capacidade de generalização, foi considerada neste teste uma condição inicial de operação diferente em relaçao ao caso base. Nesta nova condição operacional, o fluxo de potência na linha 7-8 é invertido, com a carga na barra 7 passando então a ser a maior carga do sistema. Dessa forma, o fluxo de potência será de $400 \mathrm{MW}$ no sentido da área 2 para área 1. É importante frisar que esta condiçao operacional não

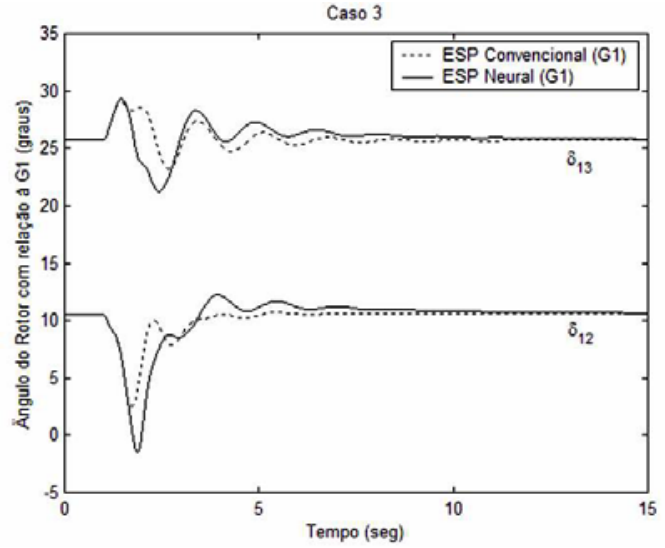

Figura 23: Resposta dos ângulos dos rotores para um curtocircuito trifásico na barra 8 , com o sistema operando na condição operacional base.

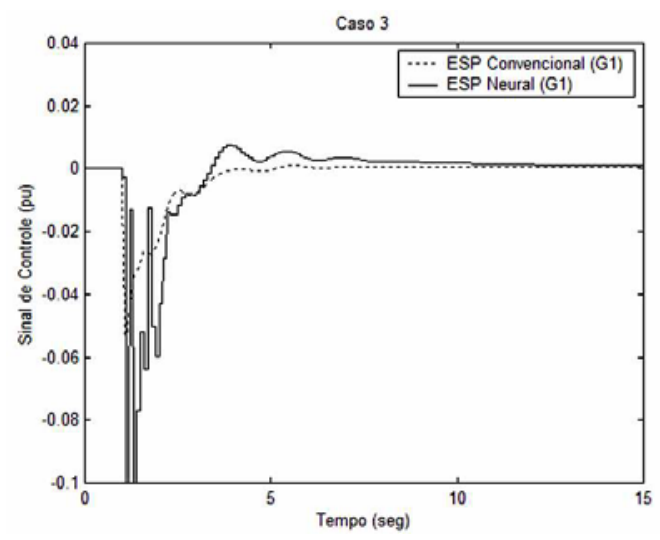

Figura 24: Comparação entre os esforços de controle do ESP neural e do ESP convencional em G1.

foi usada no treinamento do ESP neural.

Os resultados para este teste estão apresentados nas Figuras 25 e 26. A falta aplicada foi um curto-circuito trifásico na barra 8 , em $t=1,0 \mathrm{~s}$, com uma duração de $0,1 \mathrm{~s}$ e sem perda de linha, após ter sido sanado o curto-circuito. Conforme pode ser observado na Figura 25, o desempenho do sistema, quando o mesmo está equipado com ESP neural na máquina $\mathrm{G} 1$, é bastante satisfatório em relação ao amortecimento dos modos eletromecânicos do sistema. Observa-se, ainda, que a capacidade de amortecimento do modo interárea mostra-se ligeiramente degradada, quando se utiliza ESPs convencionais nos geradores. Isto deve-se ao fato de que os parâmetros dos ESPs convencionais permaneceram fixos nos valores projetados para a condiçao base de operação, a qual é bastante diferente da condição de operação utilizada neste teste. Mesmo assim, o desempenho do sistema com ESPs convencionais também poderia ser aceito como satisfatório 


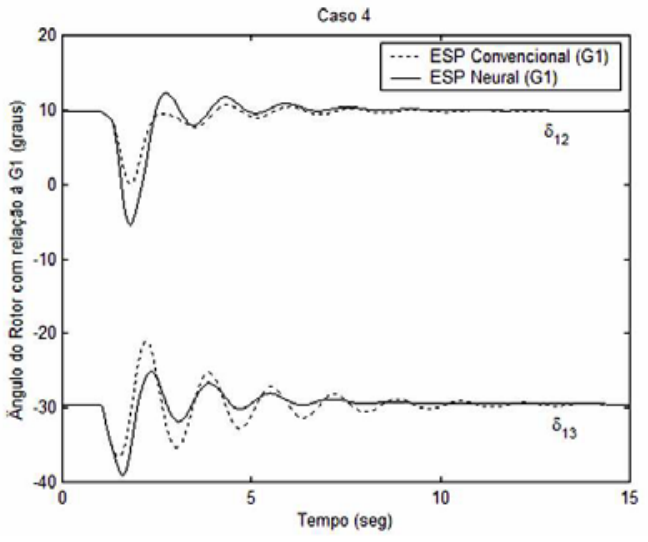

Figura 25: Resposta dos ângulos dos rotores para um curtocircuito trifásico na barra 8 , com o sistema exportando potência da área de geração 2 para a área de geração 1 .

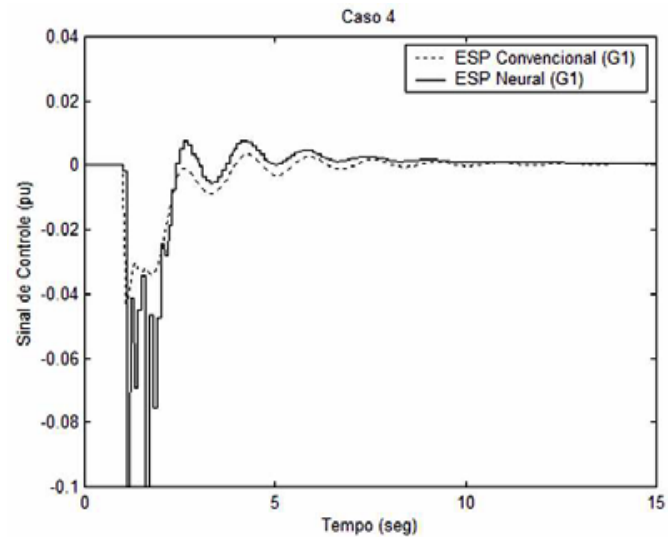

Figura 26: Comparação entre os esforços de controle do ESP neural e do ESP convencional em G1, com o sistema exportando potência da área 2 para a área 1 .

nesta condição operacional.

Na Figura 26 são apresentados os respectivos esforços de controle para o ESP neural (curva em linha cheia) e para o ESP convencional em G1 (curva em linha tracejada). Pode-se observar que os esforços de controle, para ambos os controladores, foram bastante semelhantes, mostrando assim que o bom desempenho obtido pelo ESP neural foi obtido sem ncessidade de um esforço de controle demasiadamente elevado, o que poderia interferir na magnitude da tensão terminal do gerador.

\section{CONCLUSÕES}

Um Estabilizador de Sistemas de Potência utilizando uma Rede Neural Artificial foi projetado e avaliado neste artigo.
A rede neural foi treinada com parâmetros de estabilizadores obtidos previamente, através do método de alocação de pólos para diversos pontos de operação do sistema.

Para o caso máquina síncrona - barramento infinito, a rede neural treinada apresentou, como sua característica mais importante, uma variação pequena nos parâmetros do controlador (uma região quase plana) quando o sistema opera com potência reativa positiva. Porém, estes mesmos parâmetros variam de forma bastante acentuada em pontos de operação do sistema com potência reativa negativa.

Esta propriedade leva a crer que não seria essencial utilizar ESPs com capacidade de variar seus parâmetros enquanto o gerador estivesse fornecendo potência reativa ao sistema. Apenas nos casos em que a máquina síncrona opera de forma a absorver reativo, o emprego desse tipo de estabilizador seria justificável. Testes realizados em um sistema do tipo máquina síncrona - barramento infinito demonstram que o ESP Neural proposto apresenta um desempenho muito bom, confirmando a principal característica da RNA treinada.

Conforme mostrado nos testes de simulação, a aplicação do método proposto em sistemas multimáquinas, em princípio, pode ser feita sem maiores problemas, usando um esquema de controle descentralizado, bastando que os dados para treinar cada uma das RNAs necessárias estejam disponíveis. Assim, nos locais estabelecidos de implementação de um estabilizador para amortecer determinado modo de oscilação (Martins e Lima, 1990), pode-se colocar um ESP Neural treinado com os dados obtidos de um esquema de controle a ganhos programados, usando variáveis que representem de forma adequada os diversos cenários e configurações da operação do sistema de potência considerado.

Outro ponto que deve ser enfatizado é que a rede neural, como usada neste trabalho, comporta-se como uma estrutura fixa, embora cobrindo ampla faixa de operação do sistema (resultando em superfícies não-lineares para os parâmetros do controlador). Em caso de mudanças substanciais na configuração do sistema, tería-se que retreinar a rede aqui utilizada, ou mesmo considerar-se o uso de redes neurais com memória associativa, mais indicadas para esquemas de controle adaptativo on line (Wang et al., 1995).

Todos estes tópicos citados estão sendo considerados para futuras publicações pela equipe de autores deste trabalho.

\section{AGRADECIMENTOS}

Os autores agradecem o apoio recebido da Eletronorte, através do projeto "Implementação de Estabilizadores de Sistemas de Potência em Sistemas de Excitação de Geradores Síncronos da Usina de Tucuruí, usando Técnicas Adaptativas" (ciclo 2000-2001). 
O Prof. Dr. J.A.L. Barreiros agradece também a CAPESMEC e a UFPA pelo suporte dado para a realização de estágio de Pós-Doutorado, durante dois semestres no INESC Porto (Portugal), onde este artigo foi finalizado.

\section{REFERÊNCIAS}

Acha, E., V.G. Agelidis, O.Anaya-Lara e T.J.E.Mille (2002). Power Electronic Control in Electrical Systems. Newnes Power Engineering series.

Arrilaga, J., C.P. Arnold e B.J. Harker (1983). Computer Modeling of Electrical Power Systems. John Wiley \& Sons, New York.

Aström, K.J. e P. Wittenmark (1997). Computer Controlled Systems: theory and design. $3^{R D}$ Edition. PrenticeHall, Inc., Englewood Cliffs, New Jersey.

Barreiros, J.A.L., R.R.P. Ribeiro, C.M. Affonso e E.P. Santos (1999). Estabilizador de Sistemas de Potência Adaptativo com Ganhos Programados e Rede Neural Artificial (em Espanhol). Información Tecnológica, Vol. 10, No. 4, pp. 61-65.

Cheng, S., Y.S. Chow, O.P. Malik e G.S. Hope (1986). An Adaptive Synchronous Machine Stabilizer. IEEE Trans. on Power Systems, Vol. PWRS-1, pp. 101-109.

Gu, W. e K.E. Bollinger (1989). A Self-tuning Power System Stabilizer for Wide-Range Synchronous Generator Operation. IEEE Trans. on Power Systems, Vol. 4, No. 3, pp. 1191-1199.

Guru e Hiziroglu (2001). Electric Machinery \& Transformers, $3^{r d}$ Edition. Oxford University Press, New York.

Haykin, S.S. (1998). Neural Networks: A Comprehensive Foundation. Prentice-Hall, Inc., Englewood Cliffs, New Jersey.

Hiyama, T., S. Oniki e N. Nagashima (1996). Evaluation of Advanced Fuzzy Logic PSS on Analog Network Simulator and Actual Installation on Hydro Generators. IEEE Trans. on Energy Conversion, Vol. 11, No. 1, pp. 125-131.

Hsu, Y.Y. e C.R. Chen (1991). Tuning of Power System Stabilizers Using an Artificial Neural Network. IEEE Trans. on Energy Conversion, Vol. 6, No. 4, pp. 612619.

Hunt, K.J. e T.A. Johansen (1997). Design and Analysis of Gain-Scheduled Control Using Local Controller Networks. International Journal of Control, Vol. 66, No. 5, pp. 619-651.
Kundur, P. (1994). Power System Stability and Control. McGraw-Hill, Inc., New York.

Landau, I.D. (1990). System Identification and Control Design Using P.I.M.+ Software. Prentice-Hall, Inc., Englewood Cliffs, New Jersey.

Larsen, E.V. e D.A. Swan (1981). Applying Power System Stabilizers (parts I, II, III). IEEE Trans. on Power Apparatus and Systems, Vol. PAS-100, No. 6, pp. 30173046.

Martins, N. e L.T.G. Lima (1990). Determination of Suitable Locations for Power System Stabilizers and Static Var Compensator for Damping Electromechanical Oscillations in Large Scale Power Systems. IEEE Trans. on Power Systems, Vol. PWRS-5, No. 4, pp. 1455-1469.

Mohan, N., T.M. Undeland e W.P.Robbins (2003). Power Electronics Converters, Applications and Design. $3^{r d}$ Edition. John Wiley \& Sons, Inc.

Ong, C.M. (1998). Dynamic Simulation of Electric Machinery. Prentice Hall PTR, New Jersey.

Pierre, D.A. (1987). A Perspective on Adaptive Control of Power Systems. IEEE Trans. on Power Systems, PWRS-2, pp. 387-396.

Rogers, G.J. (2000). Power System Oscillations. Kluwer Academic Press.

Shamsollahi, P. e O.P. Malik (1999). Application of Neural Adaptive Power System Stabilizer in a Multi-Machine Power System. IEEE Trans. on Energy Conversion, Vol. 14, No. 3, pp. 731-736.

Silva, A.S. e J.A.L. Barreiros (1992). Application of Adaptive Controllers to a Multimachine Power System. Proceedings of the Latincon'92 (IEEE Latin American Congress), pp. 9-13. Santiago (Chile).

Wang, H., G.P. Liu, C.J.Harris e M.Brown (1995). Advanced Adaptive Control. Pergamon. Elsevier Science Ltd.

\section{APÊNDICE}

Os parâmetros da linha de transmissão e do modelo nãolinear do gerador para o caso máquina síncrona - barramento infinito são mostrados a seguir, com reatâncias sendo expressas em pu e os outros parâmetros em segundos.

$$
\begin{aligned}
& R_{a}=0,001 ; x_{d}=1,445 ; x_{q}=0,959 ; x_{d}^{\prime}=0,316 ; x_{d}^{\prime \prime}=0,179 \\
& x_{q}^{\prime \prime}=0,162 ; R_{e}=0,02 ; X_{e}=0,415 ; T_{d o}^{\prime}=5,256 ; \\
& T_{d o}^{\prime \prime}=0,0282 ; T_{q o}^{\prime \prime}=0,157 ; H=4,27
\end{aligned}
$$

